\title{
Exploring the molecular pathways behind the effects of nutrients and dietary polyphenols on gut microbiota and intestinal permeability: a perspective on the potential of metabolomics and future clinical applications
}

Gregorio Peron ${ }^{1,2}$, Nicole Hidalgo-Liberona ${ }^{1,2}$, Raúl González-Domínguez ${ }^{1,2}$, Mar Garcia-Aloy ${ }^{1,2}$, Simone Guglielmetti ${ }^{3}$, Stefano Bernardi ${ }^{3}$, Benjamin Kirkup ${ }^{4}$, Paul Antony Kroon ${ }^{4}$, Antonio Cherubini ${ }^{5}$, Patrizia Riso ${ }^{3}$, Cristina Andrés-Lacueva 1,2,*

\section{Affiliations}

1 Biomarkers and Nutrimetabolomics Laboratory, Department of Nutrition, Food Sciences and Gastronomy, Food Technology Reference Net (XaRTA), Nutrition and Food Safety Research Institute (INSA), Faculty of Pharmacy and Food Sciences, University of Barcelona, Barcelona, Spain; gregorio.peron@ub.edu; raul.gonzalez@ub.edu; n.hidalgoliberona@ub.edu; candres@ub.edu.

${ }^{2}$ CIBER de Fragilidad y Envejecimiento Saludable (CIBERfes), Instituto de Salud Carlos III, Barcelona, Spain; gregorio.peron@ub.edu; raul.gonzalez@ub.edu; n.hidalgoliberona@ub.edu; candres@ub.edu.

${ }^{3}$ Department of Food, Environmental and Nutritional Sciences (DeFENS), Università degli Studi di Milano, Milan, Italy; patrizia.riso@unimi.it; simone.guglielmetti@unimi.it; stefano.bernardi@unimi.it.

4 Quadram Institute Bioscience, Norwich Research Park, Norwich, UK; paul.kroon@quadram.ac.uk

${ }^{5}$ Geriatria, Accettazione geriatrica e Centro di ricerca per l'invecchiamento, IRCCS INRCA, Ancona, Italy; a.cherubini@inrca.it 
*corresponding author: Prof. Cristina Andrés-Lacueva, Department of Nutrition, Food Sciences and Gastronomy; Faculty of Pharmacy and Food Sciences; University of Barcelona, 08028 Barcelona, Spain. Tel.+34 934034840, e-mail: candres@ub.edu 


\section{Abstract}

2 The gut microbiota is involved in the regulation of the intestinal permeability (IP), whose

3 disruption is a frequent condition in older people and is associated to the development of

4 several diseases. The diet can affect the gut microbiota and IP, although the molecular

5 mechanisms involved are unclear. Metabolomics is one of the suitable approaches to

6 study the effects of diet on gut microbiota and IP, although up to now the research has

7 focused only on few dietary components. The aim here was to review the most recent

8 literature concerning the application of metabolomics to the study of the diet-induced

9 alterations of gut microbiota and the effects on IP, with a particular focus on the molecular

10 pathways involved. An additional aim was to give a perspective on the future research

11 involving dietary polyphenols, because despite their potential use in the management of

12 increased IP, few studies have been reported to date.

13

14 Keywords: metabolomics, gut microbiota, intestinal permeability, nutrients, polyphenols 


\section{Introduction}

27 The gastrointestinal tract (GI) is responsible for a wide range of functions, including digestion and absorption of nutrients, water and ions, regulation of host immunity, protection against the ingress of pathogenic microorganism, and the the metabolism and detoxification of xenobiotics. The GI also hosts the largest microbial population of the human body, which works in symbiosis with the host to accomplish these various intestinal functions. Gut bacteria are particularly important for host health, being involved in the synthesis of vitamins, secondary bile acids and neurotransmitters, and playing a direct role in the metabolism and degradation of dietary components and drugs, that can affect their bioavailability and absorption ${ }^{1}$. It has been estimated that over than 1,000 different bacterial species populate the intestinal environment, with a genome comprising 100 -fold more genes than those found in human genome ${ }^{2}$. The physiological variations in the small intestine and colon, such as the presence of distinct chemical environments, nutrients and host immune activity allow distinct groups of bacterial species to populate the different regions of the lower gastrointestinal tract ${ }^{3,4}$, and this variability becomes even more complex considering the inter-individual variations and the influence of host genetics ${ }^{5-7}$. Nevertheless, most human gut microbiota share a core set of resident bacteria and related microbial genes ${ }^{8,9}$. Firmicutes, Bacteroidetes and, secondly, Actinobacteria are the three most abundant phyla, among the over 50 that have been identified by metagenomic approaches ${ }^{10,11}$. A synergistic equilibrium among the different species and the maintenance of a microbial diversity are of crucial importance for health, since the microbiota plays a central role on the proper functioning of the intestinal barrier and maintaining appropriate intestinal permeability (IP), which is directly involved in the development of numerous disorders. In this vein, a low diversity and a scarce abundance 
51 with gut disease states, e.g. Crohn's disease ${ }^{12}$, type 1 , type 2 and gestational diabetes ${ }^{13-}$ $52 \quad{ }^{15}$, celiac disease ${ }^{16}$ and obesity ${ }^{17}$.

53 Diet, as a source of macro- and micro-nutrients and other bioactive components, is one of the factors that most can affect the microbiota. Among the dietary constituents, polyphenols have been in the spotlight in recent years, due to their particular physicochemical properties and their potential to directly affect microbiota activity and host health. Polyphenols are secondary metabolites of plants, fruits and vegetables, and major components of commonly consumed foods and beverages such as chocolate, tea and coffee ${ }^{18-20}$ which, due to their characteristic (poly)hydroxylated phenyl moieties and the presence of ionizable functional groups on their scaffolds, have a low bioavailability and are scarcely absorbed by the intestine ${ }^{21,22}$. Consequently, they are prone to catabolism by the gut microbiota, which leads to the production of smaller molecular weight (MW) compounds that can be absorbed across the intestinal wall, enter the bloodstream and eventually, undergo further transformation and conjugation in the liver ${ }^{23,24}$. It has been estimated that total polyphenol absorption in the small intestine is around $5 \%-10 \%$, while the remaining $90 \%-95 \%$ transits to the large intestinal lumen and accumulates in the millimolar range ${ }^{25}$. Hence, microbial polyphenol derivatives could be responsible for the biological effects attributed to their parent compounds, or at least contribute to the overall activity. Catechins from green tea, for example, have been reported to exert antioxidant, anti-inflammatory and anti-tumorigenic activities ${ }^{26-28}$. However, the most representative green tea catechin, (-)-epigallocatechin gallate, is scarcely absorbed from the intestine and is extensively metabolized by gut microbiota ${ }^{29}$ to form smaller MW derivatives that not only contribute to the observed bioactivities of green tea, but can also exert higher activity than the parent compound ${ }^{30}$. Polyphenols and their microbial metabolites could also exert antimicrobial and bacteriostatic activities, hence regulating the overgrowth of 
harmful bacteria on the intestinal and urinary tract epithelia ${ }^{20,31}$. As an example, cranberry (Vaccinium macrocarpon Ait.) fruits, rich sources of type-A procyanidins (PAC-A), are known to exert anti-adhesive activity against the uropathogenic bacteria responsible for most of the lower urinary tract infections, although the mechanisms of action are still unknown and the outcomes of in vitro assays and in vivo clinical trials aimed at reducing urinary tract infections are frequently inconsistent ${ }^{32}$. Recent studies conducted in both rats and human volunteers show that, after supplementation with dry cranberry extracts, urine samples exert effective anti-adhesive activity against uropathogenic E. coli, despite their negligible contents of intact PAC-A ${ }^{33,34}$. However, the same urine samples were characterized by high amounts of hydroxyphenyl-valeric acid and hydroxyphenyl-valerolactone derivatives, previously reported as end-products of microbial degradation of flavan-3-ols ${ }^{35}$, indicating the important contribution of the microbial metabolites of procyanidins to the observed bioactivity ${ }^{33,34}$. Finally, the effects of polyphenols on microbiota, inflammation and oxidative stress and their capacity to regulate the synthesis and expression of specific proteins on the intestinal epithelium seem to be part of the mechanisms by which these compounds can regulate the permeability of the intestinal barrier ${ }^{36}$, whose alterations are related to the development of several diseases, especially in older subjects.

Many efforts have been made to characterize the microbial community colonizing the human intestine, for which the widespread use of metataxonomics based on 16S rRNA gene profiling and metagenomics (microbiomics) has been particularly important.

97 However, although representing powerful tools for bacterial identification and classification, microbiomics does not allow to obtain information about fluctuations in metabolic activities ${ }^{1}$. To this purpose, metabolomics is the most suitable approach, and numerous reports based on metabolomic analysis have been reported over the last decade 
$101{ }^{37}$. Focusing on the application of metabolomics in the study of diet-microbiota

102 interactions and searching for the keywords "metabolomics AND diet AND microbiota"

103 in PubMed, we found that the number of publications almost doubled from 2014 to 2018 ,

104 as an index of the popularity that metabolomics gained during the recent years (Figure 1).

105 Metabolomic approaches have been widely used to study the transformation of nutrients

106 and xenobiotics by intestinal microbiota ${ }^{38-43}$, thus allowing the characterization of

107 hundreds of metabolites derived from macro- and micronutrients and polyphenols coming

108 from fruits and vegetables. In 2009, Jacobs published a first review article regarding the

109 role of colonic microbiota in the degradation of non-digestible food ingredients and their

110 impact on gut health and immunity ${ }^{44}$. For the first time, the importance of metabolomics

111 in the study of the links between the bioconversion of non-digestible food ingredients,

112 their bioavailability and their downstream effects on microbiota composition and host

113 metabolism was recognized ${ }^{44}$. More recently, the use of integrated multi-omics

114 approaches has facilitated the study of the molecular interactions between diet and

115 microbiota, and has led to the identification of several metabolites that are produced as a

116 result of microbial metabolism of various dietary constituents. Nevertheless, considering

117 the challenges to study the mutual relationship between gut microbiota and the host, its

118 tight connection with diet, environment and lifestyle, and the still incomplete

119 characterization of the huge microbial metabolome, the path to assess precise and

120 validated metabolites to link the microbial activity to specific effects on health is just

121 starting. In a way to find a clinical relevance of metabolomics data and offer to clinicians

122 a robust tool to predict, prevent and treat several diseases, further progress is necessary.

123 The aim of this work was to review the most recent literature regarding the application of

124 metabolomics in the study of the interactions between food components and gut

125 microbiota and the effects on IP, with a particular focus on the elucidation of the 
126 molecular pathways involved. Since to date the research has mainly focused on the

127 degradation of non-digestible fibers and tryptophan and on the bioactivity of their

128 metabolites, a major part of the work will be dedicated to these important dietary

129 components. Additionally, a perspective on the future research involving the role of

130 dietary polyphenols in modulating the activity and composition of gut microbiota and the

131 effects on IP will be discussed, given that, despite their potential implication in the

132 prevention and treatment of several diseases, few clinical studies have been performed up

133 to now.

The role of microbiota and microbiota-derived dietary metabolites in regulating intestinal permeability: the application of metabolomics for the discovery of new biomarkers

138 The intestinal wall represents a barrier that selectively transports nutrients, ions and water

139 from the lumen to the bloodstream, via passive and active mechanisms. A layer of

140 epithelial cells constitutes the main physical barrier between the intestinal lumen and the

141 mucosal tissues ${ }^{45}$. Tight junctions (TJ), composed of transmembrane proteins and

142 junctional adhesion molecules that regulate the flow of water, ions and small molecules,

143 seal the paracellular spaces ${ }^{46}$. Several distinct proteins contribute to form the TJ,

144 including mainly occludins and claudins, depending on the tissue and location that

145 interlink within the paracellular space ${ }^{47}$. Although highly cross-linked, the structure of

146 TJ is dynamic, so that it can be 'opened' and 'closed' following specific stimuli ${ }^{48}$.

147 Physiological stimuli could shrink the TJ to prevent the diffusion of toxins, viruses or

148 bacterial fragments to the mucosal layer, while they can open the paracellular space to

149 allow the diffusion of nutrients ${ }^{49}$. For instance, the activation of the sodium dependent

150 glucose transporter led to the opening of TJ and allowed the diffusion of small molecules 
151 and peptides with $\mathrm{MW}<40,000 \mathrm{Da}^{50}$. On the other hand, the physiological structure and 152 dynamism of TJ could be altered due to pathological states ${ }^{51}$, leading to a condition of increased IP, also known as "leaky gut". Celiac disease, inflammatory bowel disease and

154 type I diabetes are three of the principal pathological causes of leaky gut ${ }^{52}$, which leads 155 to the permeation of potentially harmful molecules, organisms or microbial fragments 156 from the intestinal lumen to the mucosal layer, inducing a cascade of events that result in 157 immune activation and local or systemic inflammation. Older people are frequently 158 affected by decreased intestinal barrier function and consequently leaky gut ${ }^{53}$. Among 159 the causes, the aging-related decline of immune function (namely immune-senescence), 160 the remodeling of intestinal epithelium and the alterations of gut microbiota composition 161 are thought to be the most important ones ${ }^{53-55}$. As observed in disease-associated 162 increased IP, the dysfunction of the intestinal barrier in older subjects facilitates the 163 diffusion of toxic substances or peptides and microbial fragments to the mucosal layer 164 and to the bloodstream and the triggering of a systemic inflammatory response ${ }^{56}$.

165 As previously stated, diet plays an important role in the maintenance of the gut barrier 166 integrity and is hence determinant for IP. The short-chain fatty acids (SCFAs), produced 167 by the degradation of dietary fibers by several bacteria in the gut (including Clostridium, 168 Eubacterium, and Butyrivibrio), have been the most studied microbial catabolites 169 involved in the regulation of IP to date. Among them, butyrate has been identified as a 170 marker of the positive effects of non-digestible dietary fiber consumption on microbiota 171 composition and intestinal permeability. It exerts several activities on the intestinal wall, 172 such as controlling inflammation by altering the expression of pro-inflammatory 173 cytokines ${ }^{57}$, preserving the intestinal barrier function by inducing the expression of TJ 174 proteins claudin-1 and claudin-2 58 , and modulating composition of gut microbiota by 175 inhibiting the growth of pathogenic bacteria ${ }^{59}$ (Figure 2). Food is the only source of non- 
176 digestible carbohydrates, and alterations in diet lead to variations in the production of

177 intestinal butyrate. In aged mice, the increased butyrate production after the consumption

178 of high doses of soluble fiber was associated with an induced expression of the TJ proteins

179 Tjp2 and Ffar2 and to a counterbalance of the age-related microbiota dysbiosis, with a

180 significant amelioration of the increased IP condition typical of older individuals ${ }^{60}$.

181 Similar effects of a high fiber diet were also observed in mice affected by autoimmune

182 hepatitis, characterized by an imbalance of Treg/Th17 cells and increased IP ${ }^{61}$.

183 Metabolomics analysis of feces showed increased levels of butyrate after dietary

184 intervention, and the expression of TJ proteins ZO-1, occludin and claudin-1 was induced

185 in the ileum, with consequent increased intestinal barrier function and decreased

186 translocation of bacterial components through the intestinal wall ${ }^{61}$ (Table 1). The same

187 effects were also observed in mice treated with sodium butyrate, indicating a direct

188 involvement of this bacterial metabolite in the regulation of IP ${ }^{61}$. Similar results were

189 recently reported by Fachi and coll., who showed that an inulin-enriched diet protects

190 mice from Clostridium difficile-induced colitis through the production of SCFAs ${ }^{62}$.

191 Metabolomics analysis of feces showed the increased production of butyrate, propionate

192 and acetate after dietary intervention (Table 1). Butyrate reduced the levels of pro-

193 inflammatory cytokines and increased the anti-inflammatory cytokine IL-10 in the colon

194 at the peak of infection, leading to an overall attenuation of the intestinal inflammation

195 62. Butyrate induced the expression of genes associated with claudin-1 and occludin,

196 leading to a reduction of the IP and consequently to a reduction of the microbial

197 translocation in the liver and spleen ${ }^{62}$.

198 Microbial tryptophan metabolites also play an important role in regulating barrier

199 functions and gut microbiota activity. A metabolomic approach allowed to obtain

200 preliminary elucidations about the role of tryptophan and its microbial and endogenous 
201 derivatives in the regulation of immune tolerance toward intestinal microbiota ${ }^{63}$. Starting

202 from these findings, further research has elucidated the role of other microbial-derived

203 tryptophan metabolites in the regulation of gut permeability, by direct effects on epithelial

204 cells. Venkatesh et al. showed that indole-3-propionic acid (IPA), produced by the

205 firmicute Clostridium sporogenes, regulates mucosal integrity and intestinal barrier

206 function by activating the pregnane $\mathrm{X}$ receptor (PXR) and upregulating junctional

207 protein-coding mRNAs ${ }^{64}$. More recently, Dodd et al. used an integrated targeted-

208 untargeted approach to identify 12 microbial metabolites derived from the reductive

209 activity of $C$. sporogenes on aromatic amino acids (phenylalanine, tyrosyne and

210 tryptophan), of which nine (lactate, acrylate and propionate derivatives) were reported to

211 accumulate in host plasma ${ }^{65}$. The authors particularly focused on IPA and its effects on

212 gut barrier and the mucosal immune system, and their results supported the findings of

213 Venkatesh and coll. about the PXR-mediated effect on gut permeability ${ }^{64,65}$ (Table 1). A

214 treatment with $20 \mathrm{mg} \mathrm{kg}^{-1}$ IPA for four consecutive days was shown to significantly

215 decrease the IP in HFD-fed obese T2D mice ${ }^{66}$, which, prior to treatment, were

216 characterized by higher IP and lower circulating IPA levels compared to lean animals.

217 Plasma IPA amounts were also reported to increase in obese subjects 3 months after

218 Roux-en-Y gastric bypass (RYGB) surgery ${ }^{66}$, indicating, once again, the direct

219 involvement of gut microbiota in the maintenance of the intestinal barrier functions.

220 Furthermore, results from in vitro assays reported by the same authors showed that IPA

221 could reduce the permeability of T84 cell monolayer compromised by pro-inflammatory

222 cytokines ${ }^{66}$. Other metabolites derived from the same degradation pathway of

223 tryptophan, i.e. indole (produced by Escherichia coli, Clostridium bifermentans, Proteus

224 vulgaris, Paracolobactrum coliforme, Achromobacter liquefaciens, and Bacteroides

225 spp.) ${ }^{67}$, indole-3-acetic acid (produced by C. sporogenes) and tryptamine (produced by 
C. sporogenes and Ruminococcus gnavus) ${ }^{68}$, were also reported to exert anti-

227 inflammatory activity both in the intestinal lumen and in the liver ${ }^{68,69}$, and to up-regulate

228 the expression of several proteins involved in the trans-epithelial cells linkage on the

229 intestinal wall, such as tight junction proteins TJP1, TJP3, and TJP4, and gap junction

230 proteins GJE1, GJB3, GJB4, and GJA8, among others ${ }^{67}$. A schematic resume of these

231 results is reported in Figure 2.

232 In recent years, polyphenols have been widely considered for their beneficial effects on

233 health and polyphenol-rich diets have been evaluated for the prevention of several chronic

234 diseases, ranging from metabolic disorders to inflammation and cancer. Some studies

235 have also evaluated the consumption of polyphenol-rich food for the prevention of

236 diseases associated to aging, such as cognitive impairment ${ }^{70}$ and depression ${ }^{71}$, although

237 up to now the reported effects have been inconsistent. However, numerous in vitro and

238 animal studies show that the consumption of polyphenol-rich food could positively affect

239 IP, reinforcing the barrier properties of the intestinal epithelium by direct influence on the

240 synthesis and expression of tight junction proteins ${ }^{72,73}$ or by interaction with gut

241 microbiota. As previously described, this latter is directly involved in the metabolic

242 transformation of plant polyphenols and in the production of smaller MW derivatives ${ }^{74}$,

243 which in turn contribute to the maintenance of barrier function and drives changes in gut

244 microbiome constituents ${ }^{75,76}$, with important effects for host health. However, although

245 several molecular targets of dietary polyphenols and their metabolites on the intestinal

246 epithelium have been elucidated ${ }^{77}$, it is unclear how the interaction of the same

247 compounds with gut microbiota leads to beneficial effects on the intestinal barrier. In

248 recent studies, through integrated metagenomics-metabolomics analyses of feces and

249 plasma, some authors correlated the variations of the amounts of specific gut-derived

250 metabolites to the effects of polyphenol ingestion on IP (Table 1). It was observed that a 
251 high-fat diet supplemented with $4 \% \mathrm{w} / \mathrm{w}$ powdered green tea leaves rich in flavanols leads

252 to an increased intestinal population of Akkermansia spp. after 22 weeks ${ }^{78}$, a bacterium

253 that has been implied in the maintenance of a functional intestinal barrier through the

254 preservation of mucus layer thickness ${ }^{79}$. Li et al. reported that the consumption of a 255 medium-dose (20 mg/kg per day) of bilberry anthocyanin extract (BAE) promoted the 256 generation of SCFAs (acetic acid, propionic acid and butyric acid) in aging rats, through 257 the regulation of the intestinal microbiota ${ }^{80}$. Specifically, several starch-utilizing and 258 butyrate-producing bacteria (among whom Lactobacillus and Bacteroides) were induced 259 by BAE, while harmful species such as Verrucomicrobia and Euryarchaeota where 260 inhibited. These variations, associated with decreased levels of TNF- $\alpha$ and IL-6 in the 261 colon induced by BAE consumption, contributed to the restoring of the intestinal barrier 262 function typically altered in older individual ${ }^{80}$. In a more recent work by Nieman and 263 coll., the authors observed the effects of the association of acute moderate physical 264 activity (sustained walking for $45 \mathrm{~min}$ and moderate-intensity running for $2.5 \mathrm{~h}$ ) and a 265 two-week flavonoid supplementation on the IP in healthy volunteers ${ }^{81}$. The results, 266 obtained using a targeted metabolomics approach, showed that acute moderate exercise 267 leads to higher circulating amounts of 15 metabolites derived from flavonoids metabolism 268 by gut microbiota (mainly hippuric acid, methoxybenzoic acid and benzaldehyde 269 derivatives; Table 1). The increased levels of these compounds were correlated to the 270 significant decrease of IP observed in both "walking" and "running" groups of volunteers, 271 although information about the mechanism(s) of action involved are lacking ${ }^{81}$.

272 Overall, the data published up to now indicate that the effects of polyphenols on IP are 273 related to both direct activity on the expression of TJ proteins and to changes induced to 274 the intestinal microbiota, with an increase in the prevalence of species that can preserve 275 barrier functions through the production of active metabolites or by direct action on the 
mucous layer (Figure 2). On the other hand, the data supporting these observations are

277 still scarce, and up to now only few compounds (e.g. butyrate and some gut-derived 278 polyphenol metabolites) correlating the polyphenol-induced modifications of gut microbiota to the effects on the intestinal integrity and permeability have been discovered.

280 Nevertheless, as demonstrated by the works of $\mathrm{Li}^{80}$ and Nieman ${ }^{81}$, the integration of 281 metagenomics and metabolomics approaches for the study of the bacterial and metabolic composition of feces and biological fluids represents one of the most suitable approaches for the identification of the pathways leading to the effects of polyphenols on gut microbiota and IP, as well as for the assessment of the key metabolites involved.

\section{Conclusion and future perspective}

Although the study of the effects of dietary interventions on gut microbiota and IP and investigations of the mechanisms of action have begun only recently, it appears clear that appropriate dietary habits and the regular consumption of vegetables and fruits rich in fibers and polyphenols play an important role in the maintenance of proper intestinal functions. The precursors of SCFAs and of several indole or phenolic derivatives produced by bacterial catabolism in the intestinal lumen, for example, are abundant constituents of both plant-derived foods, as cereals, nuts, fruits and vegetables rich in nondigestible fibers ${ }^{82}$, and animal-based foods such as dairy products, eggs and meat, which are rich sources of tryptophan ${ }^{83}$. Thanks to the employment of integrated multi-omics approaches, the involvement of several partners (food components, microbiota and microbial-derived compounds) in the maintenance of the intestinal barrier function and the molecular pathways behind this activity are being gradually elucidated, although further efforts are required to link specific food components and their metabolites to specific mechanisms of action. Nevertheless, the increasing amounts of data regarding 
301 specific metabolites (e.g. physicochemical properties, spectroscopic properties, location 302 in biofluids, involvement in metabolic pathways) stored in freely available databases and 303 the affordability of even more sensitive and robust instrumentations will allow, in the near

304 future, to obtain further biological information to better understand the molecular 305 mechanisms behind the effects of diet on gut microbiota and IP. Once that both 306 metabolites and molecular pathways will be assessed and validated for clinical relevance, 307 they will represent novel instruments available to clinicians for the assessment of the 308 "intestinal health" and for the development of dietary plans aimed at managing and 309 preventing diseases directly linked to increased IP, as chronic inflammation and 310 immunological disorders, which are determinant for the gradual decline of health in older 311 subjects.

\section{Funding}

314 This work was supported by the Spanish Ministry of the Economy and Competitiveness 315 (MINECO) together with the Joint Programming Initiative "A Healthy Diet for a Healthy 316 Life" (JPI HDHL, http://www.healthydietforhealthylife.eu) under the Intestinal317 Microbiomics call (2015), Grant MAPLE-PCIN-2015-238 and the respective Research 318 Councils: Mipaaf (Italy; D.M. 8245/7303/2016), the Biotechnology and Biological 319 Sciences Research Council (UK; Grant BB/R012512/1). MAPLE "Gut and blood 320 microbiomics for studying the effect of a polyphenol-rich dietary pattern on intestinal 321 permeability in the elderly" was funded Funds from CIBERFES [co-funded by the

322 European Regional Development Fund (FEDER) program from the European Union 323 (EU)]. The authors also gratefully acknowledge the award from the Generalitat de 324 Catalunya's Agency for Management of University and Research Grants (AGAUR, Grant 325 2017SGR1566). RGD thanks the "Juan de la Cierva" program from MINECO (FJCI- 
2015-26590). CA-L gratefully acknowledges the financial support by ICREA under the

327 ICREA Academia programme.

328

\section{References}

330

1. Rowland, I.; Gibson, G.; Heinken, A.; Scott, K.; Swann, J.; Thiele, I.; Tuohy, K. Gut microbiota functions: metabolism of nutrients and other food components. Eur. J. Nutr. 2018, 57, 1-24.

2. Guinane, C.M.; Cotter, P.D. Role of the gut microbiota in health and chronic gastrointestinal disease: Understanding a hidden metabolic organ. Ther. Adv. Gastroenterol. 2013, 6, 295-308.

3. Donaldson, G.P.; Lee, S.M.; Mazmanian, S.K. Gut biogeography of the bacterial microbiota. Nat. Rev. Microbiol. 2015, 14, 20-32.

4. Hillman, E.T.; Lu, H.; Yao, T.; Nakatsu, C.H. Microbial ecology along the gastrointestinal tract. Microbes Environ. 2017, 32, 300-313.

5. Grice, E.A.; Segre, J.A. The human microbiome: Our second genome. Annu. Rev. Genomics Hum. Genet. 2012, 13, 151-170.

6. Ussar, S.; Fujisaka, S.; Kahn, C.R. Interactions between host genetics and gut microbiome in diabetes and metabolic syndrome. Mol. Metab. 2016, 5, 795-803.

7. Hornung, B.; Martins dos Santos, V.A.P.; Smidt, H.; Schaap, P.J. Studying

8. Turnbaugh, P.J.; Gordon, J.I. The core gut microbiome, energy balance and obesity. J. Physiol. 2009, 587, 4153-4158. 
9. Thursby, E.; Juge, N. Introduction to the human gut microbiota. Biochem. J. 2017, 474, 1823-1836.

10. Turnbaugh, P.J.; Hamady, M.; Yatsunenko, T.; Cantarel, B.L.; Duncan, A.; Ley, 
L.; Bo, S. Changes in the gut microbiota composition during pregnancy in patients with gestational diabetes mellitus (GDM). Sci. Rep. 2018, 8, 12216.

16. Nylund, L.; Kaukinen, K.; Lindfors, K. The microbiota as a component of the celiac disease and non-celiac gluten sensitivity. Clin. Nutr. Exp 2016, 6, 17-24.

17. López-Contreras, B.E.; Morán-Ramos, S.; Villarruel-Vázquez, R.; MacíasKauffer, L.; Villamil-Ramírez, H.; León-Mimila, P.; Vega-Badillo, J.; SánchezMuñoz, F.; Llanos-Moreno, L.E.; Canizalez-Román, A.; del Río-Navarro, B.; Ibarra-González, I.; Vela-Amieva, M.; Villarreal-Molina, T.; Ochoa-Leyva, A.; Aguilar-Salinas, C.A.; Canizales-Quinteros, S. Composition of gut microbiota in obese and normal-weight Mexican school-age children and its association with metabolic traits. Pediatr. Obes. 2018, 13, 381-388.

18. Manach, C.; Scalbert, A.; Morand, C.; Rémésy, C.; Jiménez, L. Polyphenols: Food sources and bioavailability. Am. J. Clin. Nutr. 2004, 79, 727-747.

19. Pandey, K.B.; Rizvi, S.I. Plant polyphenols as dietary antioxidants in human health and disease. Oxidative Med. Cell. Longevity 2009, 2, 270-278.

20. Ozdal, T.; Sela, D.A.; Xiao, J.; Boyacioglu, D.; Chen, F.; Capanoglu, E. The reciprocal interactions between polyphenols and gut microbiota and effects on bioaccessibility. Nutrients 2016, 8,78 .

21. Scalbert, A.; Morand, C.; Manach, C.; Rémésy, C. Absorption and metabolism of polyphenols in the gut and impact on health. Biomed. Pharmacother. 2002, 56, 276-282.

22. Del Rio, D.; Rodriguez-Mateos, A.; Spencer, J.P.E.; Tognolini, M.; Borges, G.; Crozier, A. Dietary (poly)phenolics in human health: Structures, bioavailability, and evidence of protective effects against chronic diseases. Antioxid. Redox Signal. 2013, 18, 1818-1892. 
23. Rechner, A.R.; Kuhnle, G.; Bremner, P.; Hubbard, G.P.; Moore, K.P.; RiceEvans, C.A. The metabolic fate of dietary polyphenols in humans. Free Radic. Biol. Med. 2002, 33, 220-235.

24. Silberberg, M.; Morand, C.; Mathevon, T.; Besson, C.; Manach, C.; Scalbert, A.; Remesy, C. The bioavailability of polyphenols is highly governed by the capacity of the intestine and of the liver to secrete conjugated metabolites. Eur. J. Nutr. 2006, 45, 88-96.

25. Clifford, M.N. Diet-derived phenols in plasma and tissues and their implications for health. Planta Med. 2004, 70, 1103-1114.

26. Yang, F.; De Villiers, W.J.S.; McClain, C.J.; Varilek, G.W. Green tea polyphenols block endotoxin-induced tumor necrosis factor- production and lethality in a murine model. J. Nutr. 1998, 128, 2334-2340.

27. Katiyar, S.K.; Matsui, M.S.; Elmets, C.A.; Mukhtar, H. Polyphenolic Antioxidant (-)-Epigallocatechin-3-Gallate from Green Tea Reduces UVB-Induced Inflammatory Responses and Infiltration of Leukocytes in Human Skin. Photochem. Photobiol. 1999, 69, 148-153.

28. Babu, P.V.A.; Liu, D. Green tea catechins and cardiovascular health: An update. Curr. Med. Chem. 2008, 15, 1840-1850.

29. Chen, H.; Sang, S. Biotransformation of tea polyphenols by gut microbiota. $J$. Funct. Foods 2014, 7, 26-42.

30. Chen, B.; Zhou, J.; Meng, Q.; Zhang, Y.; Zhang, S.; Zhang, L. Comparative analysis of fecal phenolic content between normal and obese rats after oral administration of tea polyphenols. Food. Funct. 2018, 9, 4858-4864. 
31. Marín, L.; Miguélez, E.M.; Villar, C.J.; Lombó, F. Bioavailability of dietary polyphenols and gut microbiota metabolism: Antimicrobial properties. BioMed Res. Int. 2015, 2015, 905215.

32. Fu, Z.; Liska, D.; Talan, D.; Chung, M. Cranberry Reduces the Risk of Urinary Tract Infection Recurrence in Otherwise Healthy Women: A Systematic Review and Meta-Analysis. J Nutr. 2017, 147, 2282-2288.

33. Peron, G.; Pellizzaro, A.; Brun, P.; Schievano, E.; Mammi, S.; Sut, S.; Castagliuolo, I.; Dall'Acqua, S. Antiadhesive Activity and Metabolomics Analysis of Rat Urine after Cranberry (Vaccinium macrocarpon Aiton) Administration. J. Agric. Food Chem. 2017, 65, 5657-5667.

34. Peron, G.; Sut, S.; Pellizzaro, A.; Brun, P.; Voinovich, D.; Castagliuolo, I.; Dall'Acqua, S. The antiadhesive activity of cranberry phytocomplex studied by metabolomics: Intestinal PAC-A metabolites but not intact PAC-A are identified as markers in active urines against uropathogenic Escherichia coli. Fitoterapia 2017, 122, 67-75.

35. Monagas, M.; Urpi-Sarda, M.; Sánchez-Patán, F.; Llorach, R.; Garrido, I.; Gómez-Cordovés, C.; Andres-Lacueva, C.; Bartolomé, B. Insights into the metabolism and microbial biotransformation of dietary flavan-3-ols and the bioactivity of their metabolites. Food. Funct. 2010, 1, 233-253.

36. De Santis, S.; Cavalcanti, E.; Mastronardi, M.; Jirillo, E.; Chieppa, M. Nutritional keys for intestinal barrier modulation. Front. Immunol. 2015, 6, 612.

37. Chen, M.X.; Wang, S.Y.; Kuo, C.H.; Tsai, I.L. Metabolome analysis for investigating host-gut microbiota interactions. J. Formos. Med. Assoc. 2018, 118, S10-S22. 
38. Appeldoorn, M.M.; Vincken, J.; Aura, A.; Hollman, P.C.H.; Gruppen, H. Procyanidin dimers are metabolized by human microbiota with 2-(3,4dihydroxyphenyl)acetic acid and 5-(3,4-dihydroxyphenyl)- $\gamma$-valerolactone as the major metabolites. J. Agric. Food Chem. 2009, 57, 1084-1092.

39. Van'T Slot, G.; Mattern, W.; Rzeppa, S.; Grewe, D.; Humpf, H.-. Complex flavonoids in cocoa: Synthesis and degradation by intestinal microbiota. J. Agric. Food Chem. 2010, 58, 8879-8886.

40. Sánchez-Patán, F.; Monagas, M.; Moreno-Arribas, M.V.; Bartolomé, B. Determination of microbial phenolic acids in human faeces by UPLC-ESI-TQ MS. J. Agric. Food Chem. 2011, 59, 2241-2247.

41. Moco, S.; Martin, F.-.J.; Rezzi, S. Metabolomics view on gut microbiome modulation by polyphenol-rich foods. J. Proteome Res. 2012, 11, 4781-4790.

42. Cueva, C.; Sánchez-Patán, F.; Monagas, M.; Walton, G.E.; Gibson, G.R.; Martínimmunity. Immunology 2014, 142, 24-31. 
46. González-Mariscal, L.; Betanzos, A.; Nava, P.; Jaramillo, B.E. Tight junction proteins. Prog. Biophys. Mol. Biol. 2003, 81, 1-44.

47. Furuse, M. Molecular basis of the core structure of tight junctions. Cold Spring Harb Perspect Biol 2010, 2, a002907.

48. Yu, D.; Turner, J.R. Stimulus-induced reorganization of tight junction structure: The role of membrane traffic. Biochim. Biophys. Acta Biomembr. 2008, 1778, 709-716.

49. Steed, E.; Balda, M.S.; Matter, K. Dynamics and functions of tight junctions. Trends Cell Biol. 2010, 20, 142-149.

50. Atisook, K.; Madara, J.L. An oligopeptide permeates intestinal tight junctions at glucose-elicited dilatations. Implications for oligopeptide absorption. Gastroenterology 1991, 100, 719-724.

51. Bischoff, S.C.; Barbara, G.; Buurman, W.; Ockhuizen, T.; Schulzke, J.; Serino, M.; Tilg, H.; Watson, A.; Wells, J.M. Intestinal permeability - a new target for disease prevention and therapy. BMC Gastroenterol. 2014, 14, 189.

52. Arrieta, M.C.; Bistritz, L.; Meddings, J.B. Alterations in intestinal permeability. Gut 2006, 55, 1512-1520.

53. Tran, L.; Greenwood-Van Meerveld, B. Age-associated remodeling of the intestinal epithelial barrier. J. Gerontol. Ser. A Biol. Sci. Med. Sci. 2013, 68, $1045-$ 1056.

54. Mabbott, N.A. A breakdown in communication? Understanding the effects of aging on the human small intestine epithelium. Clin. Sci. 2015, 129, 529-531.

55. Nagpal, R.; Mainali, R.; Ahmadi, S.; Wang, S.; Singh, R.; Kavanagh, K.; Kitzman, D.W.; Kushugulova, A.; Marotta, F.; Yadav, H. Gut microbiome and 
aging: Physiological and mechanistic insights. Nutr. Heal. Aging 2018, 4, 267285.

56. Di Sabatino, A.; Lenti, M.V.; Cammalleri, L.; Corazza, G.R.; Pilotto, A. Frailty and the gut. Dig. Liver Dis. 2018, 50, 533-541.

57. Canani, R.B.; Costanzo, M.D.; Leone, L.; Pedata, M.; Meli, R.; Calignano, A. Potential beneficial effects of butyrate in intestinal and extraintestinal diseases. World J. Gastroenterol. 2011, 17, 1519-1528.

58. Plöger, S.; Stumpff, F.; Penner, G.B.; Schulzke, J.; Gäbel, G.; Martens, H.; Shen,

59. Sun, Y.; O'Riordan, M.X.D. Regulation of bacterial pathogenesis by intestinal short-chain fatty acids. Adv. Appl. Microbiol. 2013, $85,92-118$.

60. Matt, S.M.; Allen, J.M.; Lawson, M.A.; Mailing, L.J.; Woods, J.A.; Johnson,

61. Hu, E.; Chen, D.; Wu, J.; Lu, F.; Chen, L.; Zheng, M.; Li, H.; Huang, Y.; Li, J.; Jin, X.; Gong, Y.; Lin, Z.; Wang, X.; Xu, L.; Chen, Y. High fiber dietary and sodium butyrate attenuate experimental autoimmune hepatitis through regulation of immune regulatory cells and intestinal barrier. Cell. Immunol. 2018, 328, 2432.

62. Fachi, J.L.; Felipe, J.S.; Pral, L.P.; da Silva, B.K.; Corrêa, R.O.; de Andrade, M.C.P.; da Fonseca, D.M.; Basso, P.J.; Câmara, N.O.S.; de Sales, E.; Souza, É.L.; Dos Santos Martins, F.; Guima, S.E.S.; Thomas, A.M.; Setubal, J.C.; Magalhães, Y.T.; Forti, F.L.; Candreva, T.; Rodrigues, H.G.; de Jesus, M.B.; Consonni, S.R.; 
Farias, A.D.S.; Varga-Weisz, P.; Vinolo, M.A.R. Butyrate Protects Mice from Clostridium difficile-Induced Colitis through an HIF-1-Dependent Mechanism. Cell Rep. 2019, 27, 750-761.

63. Lin, H.; Barnett, M.P.G.; Roy, N.C.; Joyce, N.I.; Zhu, S.; Armstrong, K.; Helsby, N.A.; Ferguson, L.R.; Rowan, D.D. Metabolomic analysis identifies inflammatory and noninflammatory metabolic effects of genetic modification in a mouse model of Crohn's disease. J. Proteome Res. 2010, 9, 1965-1975.

64. Venkatesh, M.; Mukherjee, S.; Wang, H.; Li, H.; Sun, K.; Benechet, A.P.; Qiu, Z.; Maher, L.; Redinbo, M.R.; Phillips, R.S.; Fleet, J.C.; Kortagere, S.; Mukherjee, P.; Fasano, A.; Le Ven, J.; Nicholson, J.K.; Dumas, M.E.; Khanna, K.M.; Mani, S. Symbiotic bacterial metabolites regulate gastrointestinal barrier function via the xenobiotic sensor PXR and toll-like receptor 4. Immunity 2014, 41, 296-310. nine circulating metabolites. Nature 2017, 551, 648-652.

66. Jennis, M.; Cavanaugh, C.R.; Leo, G.C.; Mabus, J.R.; Lenhard, J.; Hornby, P.J.

65. Dodd, D.; Spitzer, M.H.; Van Treuren, W.; Merrill, B.D.; Hryckowian, A.J.; Higginbottom, S.K.; Le, A.; Cowan, T.M.; Nolan, G.P.; Fischbach, M.A.; Sonnenburg, J.L. A gut bacterial pathway metabolizes aromatic amino acids into Microbiota-derived tryptophan indoles increase after gastric bypass surgery and reduce intestinal permeability in vitro and in vivo. Neurogastroenterol. Motil. 2018, 30, e13178.

67. Bansal, T.; Alaniz, R.C.; Wood, T.K.; Jayaraman, A. The bacterial signal indole increases epithelial-cell tight-junction resistance and attenuates indicators of inflammation. Proc. Natl. Acad. Sci. U. S. A. 2010, 107, 228-233. 
68. Krishnan, S.; Ding, Y.; Saedi, N.; Choi, M.; Sridharan, G.V.; Sherr, D.H.; Yarmush, M.L.; Alaniz, R.C.; Jayaraman, A.; Lee, K. Gut Microbiota-Derived Tryptophan Metabolites Modulate Inflammatory Response in Hepatocytes and Macrophages. Cell Rep. 2018, 4, 1099-1111.

69. Wlodarska, M.; Luo, C.; Kolde, R.; d'Hennezel, E.; Annand, J.W.; Heim, C.E.; Krastel, P.; Schmitt, E.K.; Omar, A.S.; Creasey, E.A.; Garner, A.L.; Mohammadi, S.; O'Connell, D.J.; Abubucker, S.; Arthur, T.D.; Franzosa, E.A.; Huttenhower, C.; Murphy, L.O.; Haiser, H.J.; Vlamakis, H.; Porter, J.A.; Xavier, R.J. Indoleacrylic Acid Produced by Commensal Peptostreptococcus Species Suppresses Inflammation. Cell Host and Microbe 2017, 22, 25-37.

70. Valls-Pedret, C.; Lamuela-Raventós, R.M.; Medina-Remón, A.; Quintana, M.; Corella, D.; Pintó, X.; Martínez-González, M.A.; Estruch, R.; Ros, E. Polyphenolrich foods in the mediterranean diet are associated with better cognitive function in elderly subjects at high cardiovascular risk. J. Alzheimer's Dis. 2012, 29, 773782.

71. Chang, S.; Cassidy, A.; Willett, W.C.; Rimm, E.B.; O'Reilly, E.J.; Okereke, O.I. Dietary flavonoid intake and risk of incident depression in midlife and older women. Am. J. Clin. Nutr. 2016, 104, 704-714.

72. Amasheh, M.; Schlichter, S.; Amasheh, S.; Mankertz, J.; Zeitz, M.; Fromm, M.; Schulzke, J.D. Quercetin enhances epithelial barrier function and increases claudin-4 expression in Caco-2 cells. J. Nutr. 2008, 138, 1067-1073.

73. Carrasco-Pozo, C.; Morales, P.; Gotteland, M. Polyphenols protect the epithelial barrier function of Caco-2 cells exposed to indomethacin through the modulation of occludin and zonula occludens-1 expression. J. Agric. Food Chem. 2013, 61, $5291-5297$. 
74. Tomas-Barberan, F.A.; Selma, M.V.; Espín, J.C. Polyphenols' Gut Microbiota Metabolites: Bioactives or Biomarkers? J. Agric. Food Chem. 2018, 66, 35933594.

75. Queipo-Ortuño, M.I.; Boto-Ordóñez, M.; Murri, M.; Gomez-Zumaquero, J.M.; Clemente-Postigo, M.; Estruch, R.; Cardona Diaz, F.; Andrés-Lacueva, C.; Tinahones, F.J. Influence of red wine polyphenols and ethanol on the gut microbiota ecology and biochemical biomarkers. Am. J. Clin. Nutr. 2012, 95, $1323-1334$.

76. Filosa, S.; Di Meo, F.; Crispi, S. Polyphenols-gut microbiota interplay and brain neuromodulation. Neural. Regen. Res. 2018, 13, 2055-2059.

77. Yang, G.; Bibi, S.; Du, M.; Suzuki, T.; Zhu, M. Regulation of the intestinal tight junction by natural polyphenols: A mechanistic perspective. Crit. Rev. Food Sci. Nutr. 2017, 57, 3830-3839.

78. Axling, U.; Olsson, C.; Xu, J.; Fernandez, C.; Larsson, S.; Ström, K.; Ahrné, S.; Holm, C.; Molin, G.; Berger, K. Green tea powder and Lactobacillus plantarum affect gut microbiota, lipid metabolism and inflammation in high-fat fed C57BL/6J mice. Nutr. Metab. 2012, 9, 105.

79. Everard, A.; Belzer, C.; Geurts, L.; Ouwerkerk, J.P.; Druart, C.; Bindels, L.B.; Guiot, Y.; Derrien, M.; Muccioli, G.G.; Delzenne, N.M.; De Vos, W.M.; Cani, P.D. Cross-talk between Akkermansia muciniphila and intestinal epithelium controls diet-induced obesity. Proc. Natl. Acad. Sci. U. S. A. 2013, 110, 90669071.

80. Li, J.; Wu, T.; Li, N.; Wang, X.; Chen, G.; Lyu, X. Bilberry anthocyanin extract promotes intestinal barrier function and inhibits digestive enzyme activity by regulating the gut microbiota in aging rats. Food. Funct. 2019, 10, 333-343. 
595

596

597

598

599

600

601

602

603

604

605

606

607

608

609

81. Nieman, D.C.; Kay, C.D.; Rathore, A.S.; Grace, M.H.; Strauch, R.C.; Stephan, E.H.; Sakaguchi, C.A.; Lila, M.A. Increased Plasma Levels of Gut-Derived Phenolics Linked to Walking and Running Following Two Weeks of Flavonoid Supplementation. Nutrients 2018, 10, 1718.

82. Dhingra, D.; Michael, M.; Rajput, H.; Patil, R.T. Dietary fibre in foods: A review. J. Food Sci. Technol. 2012, 49, 255-266.

83. Friedman, M. Analysis, Nutrition, and Health Benefits of Tryptophan. Int. J. Trytophan Res. 2018, 11, 1178646918802282. 


\section{FIGURES}

\section{Figure 1}

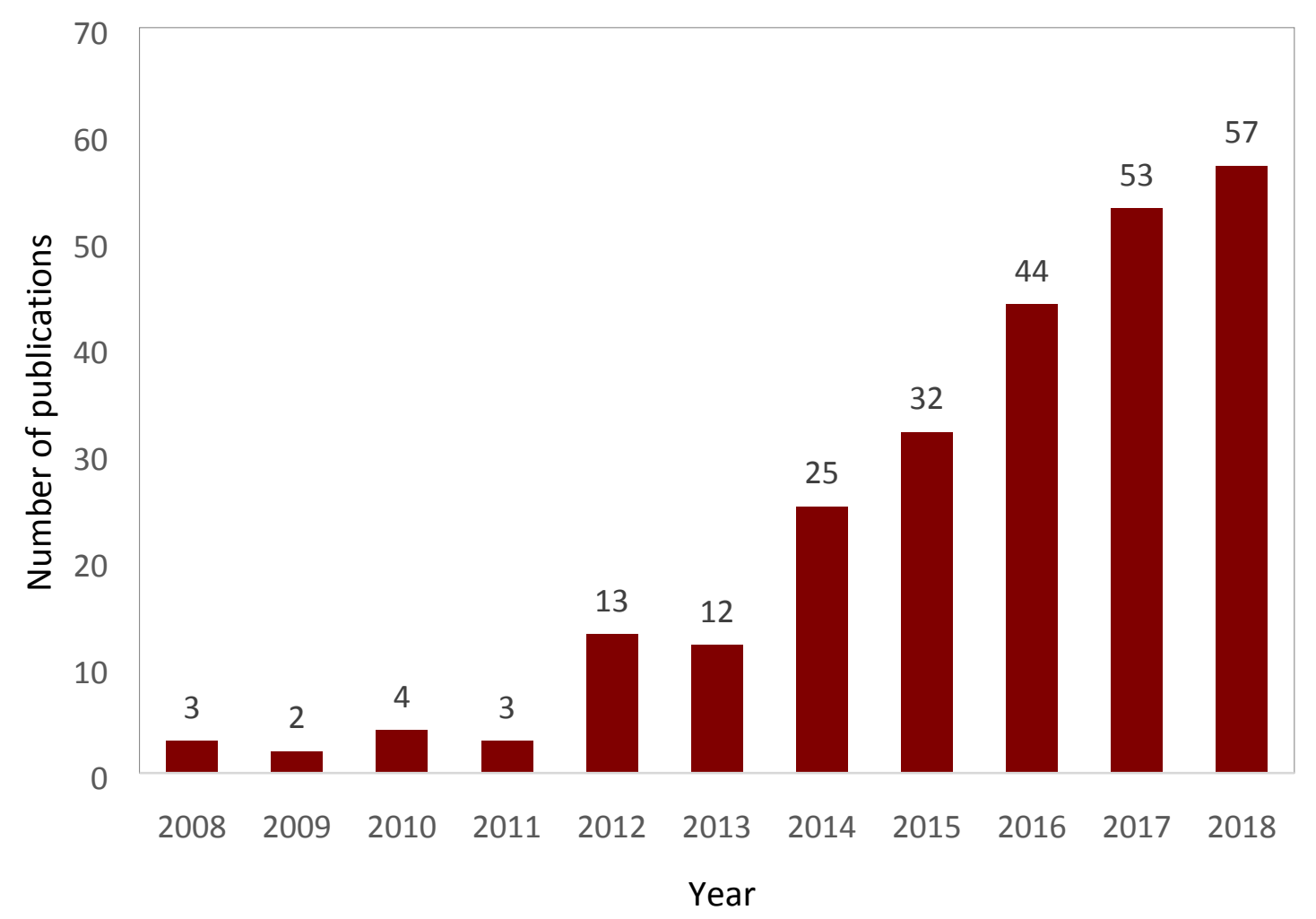




\section{Figure 2}

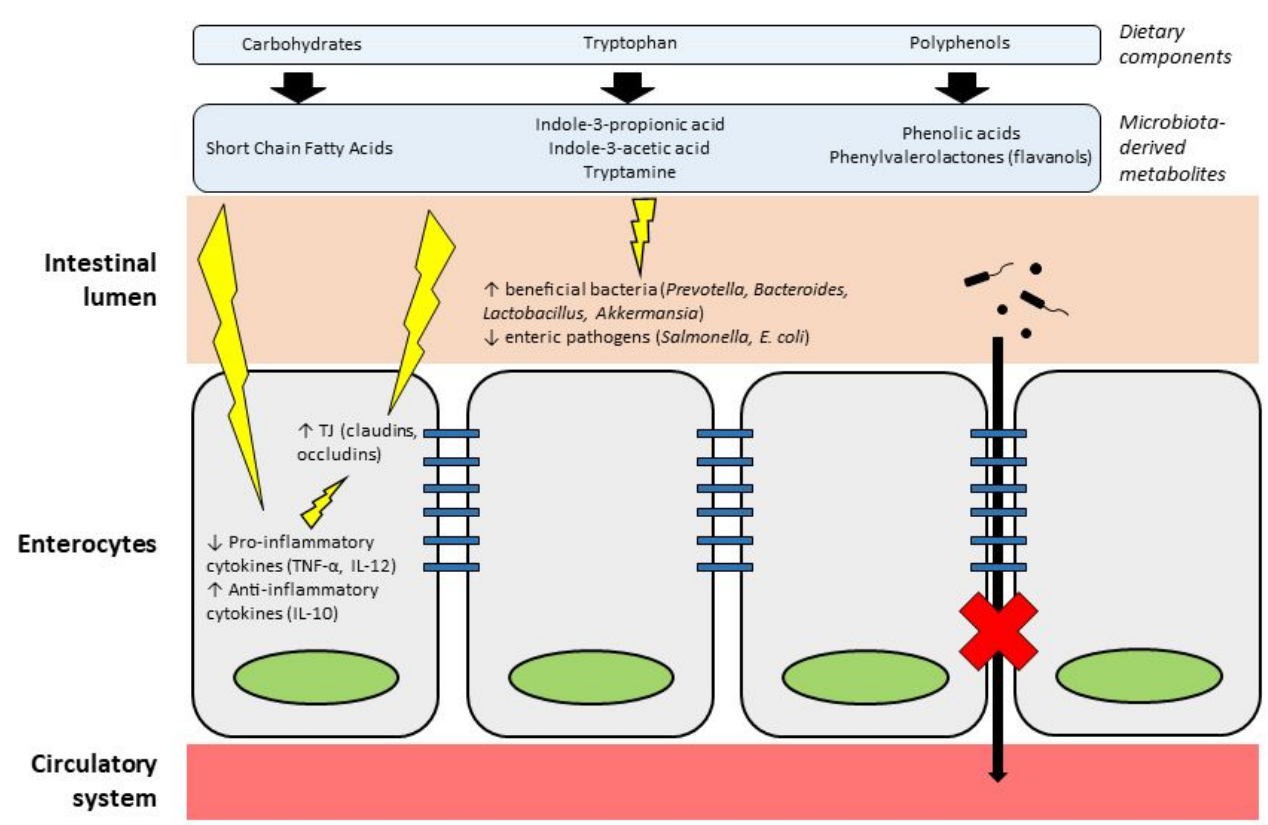




\section{TABLE}

Table 1

\begin{tabular}{|c|c|c|c|c|c|c|c|c|}
\hline & $\begin{array}{l}\text { Intervention/ } \\
\text { Condition }\end{array}$ & $\begin{array}{l}\text { Source, dose and length of } \\
\text { treatment }\end{array}$ & Model & $\begin{array}{l}\text { Biofluid/ } \\
\text { biomatrix } \\
\text { analyzed }\end{array}$ & $\begin{array}{l}\text { Metabolomic } \\
\text { approach * }\end{array}$ & $\begin{array}{l}\text { Gut-derived metabolites } \\
\text { correlated to effects on } \\
\text { IP }\end{array}$ & Main outcomes of the study ** & Reference \\
\hline \multirow[t]{2}{*}{$\begin{array}{l}\text { Dietary } \\
\text { fibers }\end{array}$} & High-fiber diet & $\begin{array}{l}\text { Laboratory diet composed } \\
\text { of } 30 \% \text { barley and } 70 \% \\
\text { standard AIN-93 for } 28 \\
\text { days }\end{array}$ & Mouse, healthy & Feces & $\begin{array}{l}\text { Targeted } \\
\text { GC-MS }\end{array}$ & $\begin{array}{l}\text { Butyrate, propionate, } \\
\text { acetate }\end{array}$ & $\begin{array}{l}\text { - Butyrate from fiber } \downarrow \text { pro-inflammatory } \\
\text { cytokines (IL-17A, IL-6, Cxcl-1) } \\
\text { - } \uparrow \text { IL-10 and TGF- } \beta \text { mRNA expression } \\
\text { - } \downarrow \text { intestinal tract lesions } \\
\text { - } \uparrow \text { Claudin-1, occludin and ZO-1 } \\
\text { - } \downarrow \text { bacterial translocation }\end{array}$ & $\begin{array}{l}\text { Hu et al., } \\
2018^{61}\end{array}$ \\
\hline & $\begin{array}{l}\text { Inulin-enriched } \\
\text { diet }\end{array}$ & $\begin{array}{l}\text { Laboratory diet } \\
\text { supplemented with } 5 \% \\
\text { cellulose and } 25 \% \text { inulin } \\
\text { for } 7 \text { days }\end{array}$ & $\begin{array}{l}\text { Mouse, healthy } \\
\text { and colonized } \\
\text { with } 1 \times 10^{8} \\
\text { CFU } \\
\text { Clostridium } \\
\text { difficile }\end{array}$ & Feces & $\begin{array}{l}\text { Targeted } \\
\text { GC-MS }\end{array}$ & $\begin{array}{l}\text { Butyrate, propionate, } \\
\text { acetate }\end{array}$ & $\begin{array}{l}\text { - Butyrate from fiber } \downarrow \text { pro-inflammatory } \\
\text { cytokines (IL-6, IL-1b, Cxcl-1) } \\
\text { - } \uparrow \text { anti-inflammatory cytokine IL-10 } \\
\text { - } \downarrow \text { intestinal tract lesions } \\
\text { - } \uparrow \text { Claudin- } 1 \text { and occludin } \\
\text { - } \downarrow \text { bacterial translocation } \\
\text { - } \uparrow \text { intestinal barrier integrity }\end{array}$ & $\begin{array}{l}\text { Fachi et al., } \\
2019^{62}\end{array}$ \\
\hline Tryptophan & $\begin{array}{l}\text { Gavage with } \\
\text { Clostridium. } \\
\text { Sporogenes and } \\
\text { standard chow } \\
\text { diet }\end{array}$ & $\begin{array}{l}\text { Standard chow (LabDiet } \\
5 \mathrm{k} 67 \text { ) containing } 0.23 \% \\
\text { tryptophan for } 4 \text { weeks }\end{array}$ & $\begin{array}{l}\text { Mouse, germ } \\
\text { free colonized } \\
\text { with } \\
\text { Clostridium. } \\
\text { sporogenes by } \\
\text { oral gavage }(\sim 1 \\
\left.\times 10^{7} \mathrm{CFU}\right)\end{array}$ & Serum & $\begin{array}{l}\text { Targeted } \\
\text { LC-MS }\end{array}$ & $\begin{array}{l}\text { Indole 3-propionic acid } \\
\text { (IPA) }\end{array}$ & $\begin{array}{l}\text { - IPA produced by C. sporogens, } \\
\text { - Colonization with C. sporogenes } \downarrow \text { intestinal } \\
\text { permeability } \\
\text { - IPA signals through PXR to fortify the } \\
\text { intestinal barrier }\end{array}$ & $\begin{array}{l}\text { Dodd eta al., } \\
2017^{65}\end{array}$ \\
\hline
\end{tabular}




$\begin{array}{lll}\begin{array}{l}\text { Gavage with } \\ \text { probiotics } \\ \text { (mice)/ }\end{array} & \begin{array}{l}\text { Oral gavage with 0.6-2 } \times \\ 10^{8} \text { CFU } \\ \text { Peptostreptococcus species }\end{array} & \begin{array}{l}\text { Mouse, dextran } \\ \text { sodium sulfate- } \\ \text { induced colitis/ }\end{array} \\ \begin{array}{l}\text { Irritable Bowel } \\ \text { Disease (IBD) } \\ \text { (human) }\end{array} & \begin{array}{l}\text { (mice) } \\ \text { Human, }\end{array} \\ & & \begin{array}{l}\text { Humer dative colitis } \\ \text { and Crohn's } \\ \text { disease }\end{array}\end{array}$

High-fat diet

(mice)

supplemented

with IPA/

Obese T2D

subjects before

and after RYGB

(human)
Daily oral gavage with 20 $\mathrm{mg} / \mathrm{kg}$ IPA for 4
consecutive days (mice)
Mouse, diet-
induced obese
(DIO)/ Human,
obese with type-

2 diabetes

$\begin{array}{ll}\text { Feces } & \begin{array}{l}\text { Untargeted } \\ \text { LC-MS }\end{array} \\ & \text { (IA) indoleacrylic acid }\end{array}$

- Peptostreptococcus species $\uparrow$ barrier

function through production of IPA and IA

- IA $\downarrow$ pro-inflammatory cytokine production

- IA $\uparrow$ intestinal epithelial barrier function

- Microbes of IBD patients have reduced

ability to cleave mucins and metabolize

tryptophan

- $\downarrow$ mucin utilization by gut bacteria in IBD

- $\downarrow$ colonization of microbes that metabolize

tryptophan in the intestine of IBD

Plasma

Targeted and

Untargeted

LC-MS,

IPA, indoxyl 3-sulfuric

- IPA $\downarrow$ IP in DIO mice

acid (ISA), indole 3 -acetic acid (IAA)

- $\downarrow$ IPA, IAA and ISA in obese subjects

- $\uparrow$ IPA, IAA and ISA 3 months after RYGB

- IPA $\downarrow$ IP in obese subjects
Wlodarska et al.,

201769

Jennis et al.,

$2018^{66}$
Rat,

young (4

months) and old

(12 months),

healthy

Human, healthy
Cecal

content

Targeted

GC-FID

10 weeks:

BBA group: $20 \mathrm{mg} / \mathrm{kg} / \mathrm{dia}$

Combination of

flavonoid

supplementatio

physical

exercise $(45$

min walking

running) total flavonoids: bilberry

anthocyanins), green tea

leaf extract (184 mg total

flavan-3-ols), $104 \mathrm{mg}$

quercetin aglycone.
Butyrate, propionate,

acetate
- $\mathrm{BA} \uparrow$ starch-utilizing and butyrate-producing

bacteria

- BA $\downarrow$ inflammatory factors (TNF- $\alpha$, IL-6)

and mucosa damages in the colon
Li et al.,

$2019^{80}$

\footnotetext{
- Physical exercise $\uparrow$ absorption of gut-derived

flavonoid metabolites

Nieman et al., $2019^{81}$

- Flavonoid consumption associated to

physical exercise $\downarrow$ IP

- Flavonoids and their gut-transformed
}

3-hydroxyhippuric acid, quercetin-3-O-

glucuronide delphinidin-

3-O-glucoside, 4-

hydroxycinnamic acid, 5- metabolites $\uparrow$ intestinal barrier integrity

(3',4'-dihydroxyphenyl)-

$\gamma$-valerolactone, 3-(3-

hydroxy-4- 
1 capsule/dia for "walking" group; 2 capsules/dia for

"running" group.

Supplementation time: 2

weeks methoxyphenyl)propanoic

acid-3-O-glucuronide

methoxybenzoic acid

derivatives, benzaldehyde

derivatives

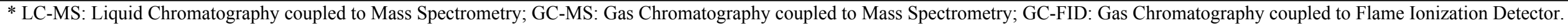

$* * \downarrow$ indicates "decrease"; $\uparrow$ indicates "increase". 


\section{FIGURE CAPTIONS}

Figure 1. The increase of the scientific literature regarding the use of metabolomics in the study of the interactions between diet and gut microbiota during the last 11 years. Source: PubMed (https://www.ncbi.nlm.nih.gov/pubmed/).

Figure 2. Schematic representation of the mechanisms of action responsible for the effects of microbiota-derived dietary metabolites on intestinal permeability.

\section{TABLE CAPTION}

Table 1. Summary of the studies involving the application of metabolomics to the study of the effects of diet-gut microbiota interactions on intestinal permeability in vivo. 


\section{GRAPHICAL ABSTRACT (TOC)}

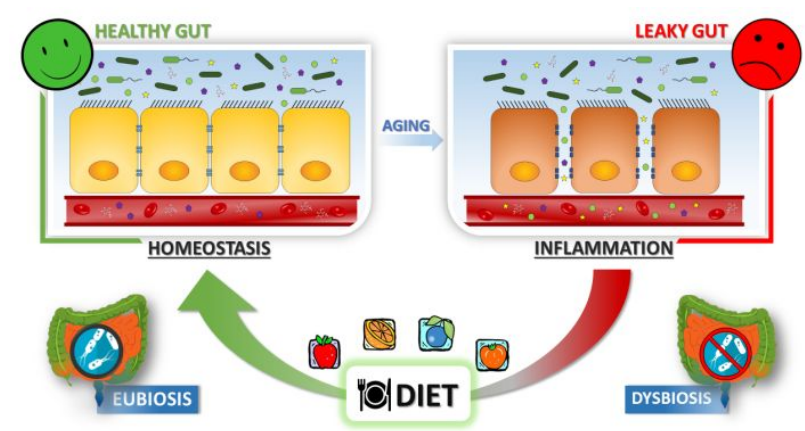

